\title{
IMPACTO DE LAS VARIABLES INDIVIDUALES EN LOS VALORES ORGANIZACIONALES DE TRABAJADORES DE EMPRESAS MANUFACTURERAS EN AGUASCALIENTES, MÉXICO
}

\author{
INDIVIDUAL VARIABLES IMPACT IN THE \\ ORGANIZATIONAL VALUES IN MANUFACTURING \\ COMPANIES EMPLOYEES LOCATED IN \\ AGUASCALIENTES, MEXICO
}

\section{Carlos Roberto Rodríguez Castellanos}

Universidad Autónoma de Aguascalientes, México

crrodri@correo.uaa.mx

\section{RESUMEN}

Se realiza una aproximación al tema de los valores organizacionales y su posible relación con las variables individuales, el estudio se aplica a trabajadores de nivel operativo de dos empresas manufactureras establecidas en el estado de Aguascalientes, México. Para la elaboración del estudio se utilizó el método del caso, con la aplicación de una encuesta a 441 personas. En general se encontró que, independientemente de las variables personales como edad, género, estado civil o antigüedad, los individuos se identifican más con el tipo de valor de apertura al cambio, lo cual habla de una adaptación al medio ambiente externo.

Palabras clave: variables individuales, valores organizacionales, empresas manufactureras

Clasificación JEL: M14 


\begin{abstract}
This research is an approach of the organizational values and their possible relationship to individual variables, this research was performed by applying a study to many employees in an operational level in two different manufacturing companies located in the state of Aguascalientes, Mexico. The method case was used in this research by applying 441 surveys. Therefore, a general finding was that in spite of personal variables such as age, gender, marital status, or years working for a company, the individuals get identified more with the type of value of openness to change in an organization, this behavior shows an adaptation to an external environment.
\end{abstract}

Key words: individual variables, organizational values, manufacturing companies.

\title{
1. INTRODUCCIÓN
}

Los valores son elementos que por lo general se incluyen en la filosofía o cultura organizacional de las empresas públicas o privadas, sin embargo, en la práctica, pocas veces se les presta atención y no es reconocida su trascendencia. Rokeach (1973: 15) establece que los grupos humanos desarrollan orientaciones normativas, es decir, concepciones de conductas preferidas y obligatorias y de situaciones deseables y no deseables, los cuales son mejor conocidos como valores.

A los valores se les define como aquellas metas deseables que pueden variar en interés para la persona o grupo y que son sus principios rectores (Schwartz: 1994, 21); por su parte Rokeach (1973: 6) establece que los valores son estándares derivados, aprendidos e internalizados de la sociedad y sus instituciones, que guían el desarrollo de una definición social personal como un miembro competente y moral de la comunidad. Los valores pregonados por alguien pueden diferenciarse de acuerdo con 
el lugar o situación en la que se encuentre, cada individuo les asigna determinada importancia o jerarquía, que dependen de sus propias vivencias, en ambos casos los valores fundamentan la actuación de las personas.

Según Rokeach (1973: 48) los valores individuales son concepciones socialmente compartidas acerca de aquello deseable y son el aprendizaje que resulta de fuerzas externas e internas que actúan sobre un individuo y moldean sus comportamientos. Schwartz (1999: 25) hace notar que los valores en la sociedad apoyan a las personas al momento de enfrentarse a las circunstancias o eventualidades que se presentan en las organizaciones en donde pasan gran parte de su vida.

En relación con los valores organizacionales, Arciniega y Zazueta (2010: 74) los definen como aquellas representaciones cognitivas de necesidades universales que se expresan a través de metas trans-situacionales que se organizan en forma jerárquica, manifestándose en el medio laboral. Debido a esto es importante considerar que los valores habrán de regular las relaciones entre los individuos y su correspondencia con organizaciones sociales como las empresas; tiene además la función principal de proveer una serie de estándares que guíen los esfuerzos para satisfacer las necesidades de los individuos.

Para una empresa, entender la influencia de los valores en el contexto de los equipos de trabajo es primordial, ya que los valores son poderosos indicadores del desempeño individual y en equipo (Glew, 2009: 671). Según Lipovetsky (1998: 258) los valores permiten a la empresa sortear ciertas problemáticas que se presentan, los valores pueden originar la "unanimidad en vez de la diversidad", la "cohesión social en vez de la oposición" o un "consenso en lugar de un conflicto"; el mismo autor también acuña un término muy importante que es el de "cooperación de todos", que consiste en estar adherido de manera colectiva y solidaria a algo en común, como los valores del grupo. En la búsqueda del consenso y correspondencia en cuanto a ciertos valores, esto no depende únicamente de las buenas intenciones de la dirección de la empresa, sino que también se requiere que un grupo de personas llegue a desarrollar lo que se conoce como "valores compartidos". 
Schwartz (1999: 25) menciona que en sociedades donde la ambición individual o el éxito son altamente valorados, la organización de la economía y sistemas legales tienden a ser competitivos; en contraste, un énfasis cultural en el bienestar del grupo tiende a ser expresado en sistemas legales y económicos más cooperativos. Finalmente, Jin y Drozdenko establecen que "Un negocio debería rápidamente posicionarse con sus propias bases, en el servicio que provee a la sociedad. Las ganancias no deberían ser el reflejo de la avaricia corporativa, sino un voto de confianza de la sociedad hacia la empresa" (2010: 342), con lo que se da a entender por qué la primacía de los valores organizacionales es fundamental para toda empresa.

Al considerar el marco de referencia anterior sobre la importancia de los valores en las organizaciones, esta investigación tiene como objetivo principal determinar si existe relación entre las variables individuales de los trabajadores y los valores organizacionales. Para la realización del objetivo se instrumentó un análisis empírico mediante el estudio de caso en dos empresas manufactureras.

Con la intención de aprehender los valores organizacionales de los trabajadores se utilizó el instrumento denominado Escala de valores hacia el trabajo (EVAT 30) de Arciniega y Zazueta (2010: 161). En dicha escala se toman en cuenta cuatro valores de orden superior: apertura al cambio, conservación, automejora y autotrascendencia. Los resultados arrojados por esta indagación son importantes para los directivos o administradores de personal, ya que pueden conocer los valores más preciados para los trabajadores, y con base en ello diseñar estrategias que les permitan promover en la organización un comportamiento que esté alineado con la tarea fundamental de la empresa.

El trabajo está organizado de la siguiente manera: después de la introducción se realiza la revisión de la literatura, posteriormente se establece la metodología, que se explica la muestra que se utilizó, además de las técnicas utilizadas para recolectar la información y medir las variables; después se analizan los resultados y finalmente se plantean las principales conclusiones a las que llevan los hallazgos. 


\section{REVISIÓN DE LA LITERATURA}

Los valores son parte fundamental de la moral, Cuevas conceptualiza la moral como "... un conjunto de deberes, de hábitos, de mandamientos; es un sistema de prohibiciones el cual tiene como finalidad contener, poner límite a las pasiones y limitar la sensibilidad de acuerdo con las órdenes de la razón...” (2005: 123). El autor refiere que los autores contemporáneos de la ética tienen la convicción de fundar la "ética de la empresa" con base en los valores de la sociedad capitalista, por lo que es común escuchar acerca de organizaciones que se consideran democráticas, justas o liberales, pero que en la práctica sus comportamientos son contrarios a lo que promueven (2005: 136). El énfasis en los valores que caracterizan a una cultura se comunica a los miembros a través de la exposición cotidiana de costumbres, leyes, normas, instrucciones y prácticas organizacionales; con lo cual se consigue que los miembros de un grupo cultural compartan ciertos valores sociales (Schwartz, 1999: 25-26).

En las organizaciones no es posible hablar de valores como libertad, igualdad o justicia sin llegar a cuestionar aspectos estructurales como el poder, la autoridad o las ganancias, es entonces que surgen otros valores importantes en sustitución de los anteriores, como el compromiso, la responsabilidad, el respeto, la honestidad o la lealtad (Rodríguez, 2012: 77). Un caso significativo respecto a la ponderación que se le da a los valores es el mencionado por Pascale y Athos (1981: 72- 74) acerca de Matsushita, empresa que infunde a sus trabajadores dos clases de entrenamiento, uno relativo a las habilidades necesarias para el trabajo y otro (para ellos el más importante), el entrenamiento acerca de los valores Matsushita. Sagiv y Schwartz (2007: 177) mencionan que en las organizaciones los símbolos y rituales expresan sus valores, los uniformes hacen notar el estatus o rango en el ejército u hospitales, mientras que uniformes idénticos utilizados en una escuela o institución pretenden ocultar diferencias socioeconómicas o jerarquías.

Schmeltz (2014: 255) realizó un estudio respecto a los valores organizacionales en diferentes empresas, en el analizó los valores de sus trabajadores desde la perspectiva de los valores corporativos y los valores 
referentes a la responsabilidad social corporativa, los cuales en teoría deberían tener correspondencia. Los resultados mostraron que no existía una correlación entre los valores corporativos y los valores enfocados a la responsabilidad social corporativa expresados por los trabajadores, a pesar de estar trabajando en una misma empresa. Schmeltz (2014: 248) justifica sus hallazgos al recordar que existen valores terminales, mismos que son deseos o estados finales trascendentes para la vida, y al haber también valores instrumentales que son comportamientos o principios para conseguir los primeros. Si se consideran los valores es claro que las empresas priorizan los valores instrumentales a expensas de los terminales, por lo cual el autor no encontró una alineación entre los valores corporativos y los valores de la responsabilidad social corporativa.

Florea, Cheung y Herndon (2013: 406) investigaron acerca del papel que ocupan los valores organizacionales en relación con la sustentabilidad organizacional, toman en cuenta cuatro valores principales: altruismo, empatía, reciprocidad y modestia, que dan soporte efectivo a las prácticas de recursos humanos. Los autores establecieron que la sustentabilidad organizacional depende de cómo son los empleados (de acuerdo con sus valores) y no únicamente de las prácticas de administración de recursos humanos.

Jintae y Xiao (2012: 2157) realizaron un estudio sobre la relación de los valores organizacionales con la satisfacción laboral y el compromiso organizacional. Los hallazgos revelaron que la satisfacción laboral actúa como mediador entre los valores y el compromiso organizacionales, lo cual se debe a que los valores tienen una influencia importante en el compromiso organizacional.

Bell-Laroche, Kerwin y MacLean (2014: 653) al analizar organizaciones deportivas no lucrativas encontraron un impacto positivo en los trabajadores al emplear una filosofía de administración por valores, por lo que los líderes de este tipo de empresas perciben una contribución que permite mejorar el desempeño organizacional.

A la par de lo anterior, se han desarrollado estudios en los que se ha evaluado la relación entre los valores y diversas variables demográficas, ya sea en individuos de la misma nacionalidad o de distintos países (Schwartz, 1994; Schwartz, 1999, Arciniega, 1999; Devos, Spini y Schwartz, 2002; 
Nelson y Gopalan, 2003; Alducin, 2004; Sagiv y Schwartz, 2007; Glew, 2009; Stelzl y Seligman, 2009; Jin y Drozdenko, 2010). El estudio de los valores se ha abordado desde distintas perspectivas, Schwartz (1994) elaboró y coordinó un estudio en el que se distinguieron diez tipos de valores principales como motivación de las personas y se llevó a cabo en 44 países. Entre los diez valores principales que halló Schwartz (1994: 22) se encuentran: poder, logro, hedonismo, estimulación, auto dirección, universalismo, benevolencia, tradición, conformidad o seguridad. Schwartz (1999: 25) menciona que la forma en la que las instituciones sociales actúan, sus objetivos y su modo de operación expresan sus prioridades de valores; por ejemplo, en sociedades donde la ambición individual o éxito son ampliamente valorados, la organización de la economía y sistemas legales tienden a ser competitivos, mientras que en sociedades donde el bienestar del grupo es importante, esto se expresa mediante sistemas económicos y legales más cooperativos.

Arciniega (1999) estudió los valores hacia el trabajo en ocho ciudades de México, mostró la diversa conceptualización de esta variable en los distintos lugares donde se aplicó el instrumento, por lo que concluyó que, a pesar de la creencia generalizada de una cultura homogénea en un país, los resultados fueron heterogéneos de acuerdo con el género, religión o lugar de residencia. Respecto a la relación entre los valores y la religión, Arciniega (1999: 3) encontró que valores relacionados con la conformidad, como la obediencia o el respeto, fueron clasificados más alto en ciudades del noroeste de México, en tanto que valores como la constancia, recibieron una mayor ponderación en las ciudades de León, Guadalajara o Puebla, mismas que se caracterizan por una mayor proporción de católicos practicantes. Por su parte Devos, Spini y Schwartz, (2002: 488) observaron que las personas católicas expresan una fuerte adhesión a los valores de conservación.

En el caso del comportamiento de los valores a través de diversas culturas, Stelzl y Seligman (2009: 966) estudiaron las diferencias entre los valores de individuos con culturas distintas (oriental y occidental) y encontraron que mientras que para las personas de origen asiático el valor más importante era el de conformidad (conservación), para occidentales, 
como los canadienses, era el de benevolencia (autotrascendencia). Alducin (2004: 38) al estudiar los valores de los mexicanos, observó que aquellos con los que más se identificaban eran, por ejemplo, ser trabajador, religioso, patriota, alegre o aguantador (tolerante).

Sagiv y Schwartz (2007: 187), analizaron cómo los valores de la sociedad afectan los valores de la organización y encontraron que las variables que más influyen son los valores sociales y personales, además de la naturaleza de las tareas que realizan en la organización. Glew (2009: 670) realizó un estudio en el que comparó a estudiantes de nivel licenciatura y maestría para conocer la influencia de los valores personales en el desempeño individual y de equipo; debe precisarse que los resultados no fueron significativamente diferentes en los niveles educativos. La investigación descubrió que, en el nivel individual, los estudiantes le dieron mayor importancia al valor de logro pero, inesperadamente, al hablar de la evaluación en grupo, la ponderación fue inversa; además el autor estableció que a nivel grupal los miembros del equipo le dieron mayor calificación al valor de igualdad, que a nivel individual.

Una aportación sumamente interesante es la que realizan Jin y Drozdenko (2010: 345), ellos llevan a cabo una investigación acerca de la actitud de los individuos hacia los valores en organizaciones orgánicas ${ }^{1}$ o en mecanicistas. ${ }^{2}$ Los autores concluyeron que las actitudes de los administradores hacia los valores y la responsabilidad social están relacionados con los valores orgánicos y mecanicistas de las empresas a las que pertenecen. En tanto que en organizaciones con valores orgánicos hay una mayor identificación con la responsabilidad social, en aquellas con

1 La organización orgánica se caracteriza por una estructura plana, con comunicación e interacción horizontal, una baja especialización, ya que el conocimiento está en todos lados, y por la descentralización, debido a que las decisiones son tomadas con la participación de la mayoría. Este tipo de organizaciones, comparativamente, son más complejas de formar, pero más adaptables, flexibles y sustentables en ambientes donde el cambio es veloz e impredecible (consultado el 21 de enero de 2013 en: http://www.businessdictionary.com/definition/organic-organization. html\#ixzz2HUEx5sov).

2 La organización mecanicista es aquella con estructura jerárquica o burocrática, caracterizada por la centralización de la autoridad, la formalización de procedimientos o prácticas y la especialización de funciones; comparativamente es fácil de organizar, pero tiene dificultad para adaptarse al cambio rápidamente (consultado el 21 de enero de 2013 en: http://www.businessdictionary.com/definition/mechanistic- organization.html\#ixzz2HUEXW1Ec) 
valores mecanicistas ocurre lo contrario. Por otro lado, se demostró que al cambiar de valores organizacionales mecanicistas a orgánicos se puede influir en la ética y responsabilidad social de la organización; lo que hace pensar que los valores orgánicos facilitan y fortalecen la adopción de dichas tendencias. Según Jin y Drozdenko (2010: 356) el proponer sistemas de valores orgánicos trae consigo motivación en las organizaciones, ya que se promueve el empoderamiento, la apertura a compartir información, una ideología democrática y mayor sentido de responsabilidad social y la ética.

Por otro lado, respecto a las variables individuales, una recurrente al momento de estudiar los valores es la de edad; Abboushi (1990: 62-63) encontró diferencias significativas entre trabajadores jóvenes y maduros. En el caso de los más jóvenes, sus resultados indicaron que estos perciben el valor trabajo como origen del prestigio social, mientras que los trabajadores maduros le atribuían menos importancia a dicho valor. Arciniega (1999: 4) descubrió que los empleados mayores de 40 años le atribuyen mayor importancia a valores semejantes a la conformidad y seguridad,en comparación con individuos mayores de 30 años; por otro lado, personas entre 20 y 30 años le atribuían mayor ponderación a valores relacionados, como autodirección, hedonismo y logro.

Otra variable interesante de estudiar es el género, Arciniega (1999:4) observó que los valores asociados al logro o la auto dirección, como la creatividad o las habilidades, tienen una calificación más alta en hombres que en mujeres, mientras que valores como la lealtad e independencia tienen una mayor ponderación entre las mujeres que entre los hombres. Abbu-Saad e Isralowitz (1997: 749) realizaron un estudio en el que analizaron la relación entre el género y los valores hacia el trabajo, en estudiantes universitarios. Su indagación demostró que existían divergencias entre los valores de estudiantes de facultades, como las de ciencias sociales, ingeniería o medicina, sin embargo, esas desigualdades no mostraban patrones consistentes de los valores hacia el trabajo fundamentados en el género.

Por su parte, Posner (2010: 540) no encontró diferencias significativas entre los valores personales y organizacionales de hombres o mujeres, en tanto que en otros estudios citados por el mismo autor, sí se habían 
presentado divergencias. Posner estableció que al haber congruencia entre los valores personales y los valores organizacionales hay un beneficio tanto para los individuos como para la organización, lo que crea un lazo más fuerte, además de sentimientos afectivos que impactan en el desempeño.

En cuanto al estado civil, Abbu-Saad e Isralowitz (1997: 749) no observaron diferencias significativas de los valores al analizar su muestra total de mujeres y hombres, un comportamiento similar se presentó al examinar los valores de personas solteras y muy pocas diferencias se encontraron al observar los valores de personas casadas.

Respecto a la antigüedad en el trabajo, Abboushi (1990: 63) encontró que los trabajadores con menor tiempo laborando en la organización (hasta diez años) mostraban un interés alto por esforzarse para escalar en la jerarquía, lo que trae consigo una mejor paga y un mayor reconocimiento. Por otro lado, algo de llamar la atención, era que los trabajadores con mayor antigüedad en la organización (20 años o más) exhibían un interés moderadamente alto, lo cual probablemente se debía a que buscaban mejorar sus condiciones laborales antes de retirarse. En contraste, los trabajadores con una antigüedad media (11 a 20 años) presentaban un interés medio por ascender en la jerarquía, quizá debido a que ya habían accedido a ciertos privilegios, además de que aún veían lejano su retiro. Por lo anterior, puede considerarse que el autor descubrió que la antigüedad sí influye al momento de querer mejorar la posición jerárquica dentro de la organización, algo que concuerda con los valores de estabilidad, logro, competitividad o prestigio.

A su vez Posner (2010: 540) descubrió que los respondientes a su estudio con un mayor tiempo de laborar en una empresa mostraban un mayor alineamiento de sus valores personales con los valores de la organización. Además de lo anterior, los hallazgos de Posner establecieron que al haber una alineación entre los valores personales y organizacionales hay una diferencia real en el lugar de trabajo, ya que se contribuye a actitudes de compromiso, motivación, ética o eficacia.

Con base en la revisión de la literatura se plantean las siguientes hipótesis de investigación: 
H1: Las variables individuales de los trabajadores influyen significativamente en el tipo de valor organizacional expresado por éstos.

$\mathrm{H} 0$ : Las variables individuales de los trabajadores no influyen significativamente en el tipo de valor organizacional expresado por ellos.

Con la aceptación o rechazo de $\mathrm{H} 1$ se pretende encontrar si las variables individuales de los trabajadores, tales como edad, género, estado civil o antigüedad, actúan sobre el tipo de valor expresado por cada uno de éstos. Una vez obtenidos los resultados, se pretende compararlos con los encontrados en las indagaciones previamente mencionadas en la revisión de la literatura sobre los valores organizacionales.

\section{METODOLOGÍA}

Con el objeto de corroborar la relación entre las variables individuales y los valores organizacionales, se realizó una investigación empírica utilizando el estudio de caso. La razón por la cual se eligió el estudio de caso tiene que ver con que éste es un primer acercamiento para hacer futuras investigaciones en las que se abarquen más organizaciones; la elección del tamaño grande de las empresas se debió a que en ellas, por lo general, existe una mayor preocupación por trasmitir aspectos de la cultura organizacional, como los valores organizacionales.

\subsection{Muestra y recolección de datos}

Las empresas estudio de caso se eligieron mediante una muestra no probabilística utilizando como criterio el que las organizaciones se preocuparan por fomentar sus valores organizacionales, además de tener la apertura para indagar en las mismas. Se aplicó una encuesta personal a trabajadores de nivel operativo, puesto que es ahí donde permean la cultura y valores organizacionales promovidos por la empresa. En el cuadro 1 se presenta la información general de las empresas estudio de caso. 
Cuadro 1. Ficha técnica de la investigación

\begin{tabular}{|l|l|l|}
\hline \multicolumn{1}{|c|}{ Características } & \multicolumn{1}{c|}{ Caso 1 } & \multicolumn{1}{c|}{ Caso 2 } \\
\hline Nacionalidad & Estadounidense & Japonesa \\
\hline Población & $\begin{array}{l}1,800 \text { empleados de nivel } \\
\text { operativo }\end{array}$ & $\begin{array}{l}\text { 918 empleados de nivel } \\
\text { operativo }\end{array}$ \\
\hline Ámbito de estudio & Instalaciones de la planta & Instalaciones de la planta \\
\hline Unidad muestral & Empresa estudio de caso & Empresa estudio de caso \\
\hline Recolección de la información & Encuesta personal & Encuesta personal \\
\hline Procedimiento de muestreo & $\begin{array}{l}\text { Estudio de caso. Muestreo } \\
\text { probabilístico (MAS) }\end{array}$ & $\begin{array}{l}\text { Estudio de caso. Muestreo } \\
\text { probabilístico (MAS) }\end{array}$ \\
\hline Tamaño de la muestra & 270 trabajadores operativos & 171 trabajadores operativos \\
\hline Margen de error de muestreo & $\begin{array}{l} \pm 5.5 \% \text { con un nivel de } \\
\text { confianza de } 94.5 \%, \text { con } \mathrm{p}=0.5\end{array}$ & $\begin{array}{l} \pm 5.5 \% \text { con un nivel de } \\
\text { confianza de } 94.5 \%, \text { con } \mathrm{p}=0.21\end{array}$ \\
\hline Fecha del trabajo de campo & Mayo de 2011 & Julio de 2011 \\
\hline Paquete estadístico & SPSS 15 & SPSS 15 \\
\hline
\end{tabular}

Fuente: elaboración propia.

A continuación se realiza una descripción de las organizaciones en las cuales se llevó a cabo el estudio:

a) Caso 1, de origen estadounidense, se dedica a la fabricación de componentes electrónicos para diversas industrias, surge en 1969 en Estados Unidos y ha tenido un crecimiento constante hasta llegar a ser una de las compañías de manufactura de productos electrónicos más importante del mundo, con más de 200 mil empleados. En México existen plantas en Guadalajara, Ciudad Juárez, Tijuana y Ramos Arizpe. La planta de Aguascalientes tiene 11 años, aunque es importante precisar que el personal y los activos fijos de la empresa pertenecieron anteriormente a otra empresa que después la vendió a la primera, a principios de la década pasada. Actualmente la planta tiene más de 2 mil trabajadores y más de 20 clientes a nivel global. 
b) Caso 2, de origen japonés, se dedica a la fabricación de asientos para automóvil, cuenta con 918 trabajadores en su planta de Aguascalientes. La empresa se funda en la ciudad de Aguascalientes en 1991 y tiene plantas en Aguascalientes, Guadalajara, Cuernavaca y Zacatecas. Los principales clientes de la organización son Nissan, Honda, Kasai, Tacle y Setex. También se fabrican partes para la industria automotriz como son: vestidura, frame, doblado de tubos y alambres, espumado y ensamble. En octubre de 1996 se inició la producción de asientos en la planta de Aguascalientes para los automóviles Honda, cuya planta está localizada en Guadalajara, Jalisco.

\subsection{Medición de variables}

Para efectos de la presente investigación se desarrolló una encuesta en la que se utilizó un cuestionario que incluía cuestionamientos relacionados con variables individuales como edad, género, estado civil y antigüedad en el trabajo. La elección de las variables individuales se debió a que estas mismas ya habían sido analizadas en estudios previamente citados. A la par de lo anterior y, con la intención de analizar los valores organizacionales, se utilizaron preguntas provenientes del cuestionario denominado Escala de valores hacia el trabajo (EVAT 30, en Arciniega y Zazueta, 2010), que consta de 16 ítems, los cuales operacionalizan cuatro valores de orden superior (gráfica 1), cada ítem tiene siete opciones que van desde "es totalmente distinto a mí" hasta "es idéntico a mí". Dicha escala con cuatro valores de orden superior ha sido aplicada con éxito en distintos estudios realizados tanto dentro como fuera de México, por lo que se le considera apropiada para estos casos en particular. A continuación se especifican cada uno de los tipos de valores:

1. Apertura al cambio. Se da en una organización enfocada al exterior con un alto grado de flexibilidad e individualidad que busca la innovación, la independencia y la creatividad. 
2. Automejora. Se da en el enfoque hacia la posición externa, con la necesidad de estabilidad y control, el logro, la competitividad y prestigio.

3. Autotrascendencia. Se enfocan al ambiente interno mediante flexibilidad, preocupación por la gente y los clientes; manifestándose la tolerancia, el bienestar o la solidaridad.

4. Conservación. Una organización con enfoque hacia el interior, necesidad de estabilidad y control busca respeto, aceptación y tradición (Arciniega y Zazueta, 2010: 63-64).

En el cuestionario se solicita a los encuestados que se evalúen y comparen si son semejantes con los empleados que se describen, empleando respuestas tipo Likert.

\section{Gráfica 1. Estructura dinámica de los valores}

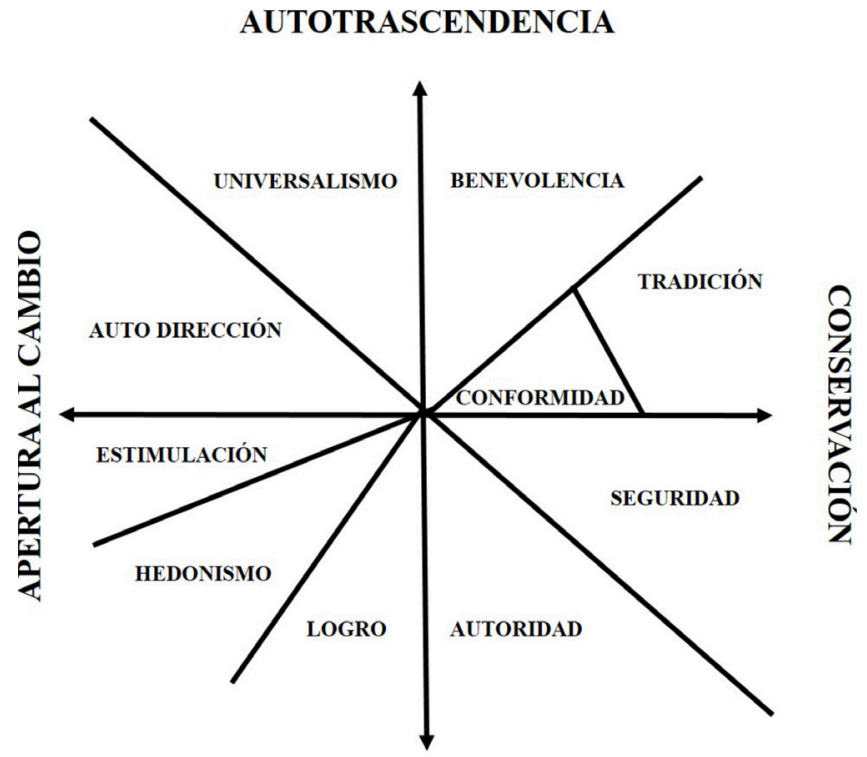

AUTOMEJORA

Fuente: Schwartz en Arciniega y Zazueta (2010: 63). 


\section{ANÁLISIS DE RESULTADOS}

\subsection{Análisis univariante}

En primer lugar se muestran los estadísticos descriptivos para las puntuaciones de encuestados de los dos casos, respecto a las cuatro tipologías de valores organizacionales (cuadro 2), se ordena la clasificación con base en las medias observadas, acerca de la importancia relativa que los trabajadores dan a determinado valor organizacional.

Cuadro 2. Estadísticos descriptivos de los valores organizacionales

\begin{tabular}{lcccc}
\hline \multicolumn{1}{c}{ Tipología de valor } & Media & Media (\%) & Desv. est. & Rango \\
\hline Auto trascendencia & 5.23 & 74.8 & 1.13 & $1.75-7$ \\
Apertura al cambio & 4.83 & 69.1 & 1.10 & $1-7$ \\
Conservación & 3.88 & 55.5 & 0.96 & $1.5-7$ \\
Auto mejora & 3.45 & 49.4 & 1.22 & $1-7$ \\
\hline
\end{tabular}

Fuente: elaboración propia.

En este análisis inicial puede observarse que los trabajadores otorgan una mayor ponderación al tipo de valor organizacional de auto trascendencia. Dicho valor busca una estabilidad en las relaciones dentro de la empresa por medio de la flexibilidad y un buen trato hacia la gente, comparten valores organizacionales relacionados con el respeto, bienestar o compañerismo (Arciniega y Zazueta, 2010: 63-64). Estos valores son muy apreciados por la mayoría de las personas, sin embargo, no en todos los casos coinciden con la forma de trabajo desarrollada en una empresa, debido a los múltiples compromisos y responsabilidades de ésta. 
Posteriormente se analizó la tipología de valores organizacionales que tenía un predominio en las empresas estudio de caso (cuadro 3), se encontraron coincidencias en cuanto al orden que los trabajadores dieron en ambas organizaciones a los cuatro principales valores.

Cuadro 3. Tipología de valores organizacionales

\begin{tabular}{lcc}
\hline \multicolumn{1}{c}{ Valor } & Caso 1 & Caso 2 \\
\hline Auto trascendencia & 5.22 & 5.25 \\
Apertura al cambio & 4.91 & 4.69 \\
Conservación & 3.89 & 3.86 \\
Auto mejora & 3.49 & 3.43 \\
\hline
\end{tabular}

Fuente: elaboración propia.

En general se puede apreciar una preponderancia del valor de auto trascendencia (cuadro 3), que tiene que ver con el universalismo o la benevolencia y que se relaciona con valores como el aprecio, la tolerancia y la protección del bienestar de las personas y de la naturaleza (Schwartz, Ros y Surkiss, 1999: 52-53). El valor con una menor ponderación es el de auto mejora, el cual se enfoca a los valores de logro o autoridad, que están relacionados con el éxito social por medio de la competencia o consecución de estatus y prestigio, además de control y dominio de las demás personas y de los recursos (Schwartz et al., 1999: 52-53). A continuación se muestran, mediante un esquema (gráfica 2), los dos casos analizados y las tendencias en cuanto a las preferencias en los valores organizacionales, apreciándose claramente la propensión hacia la auto trascendencia y la oposición a la auto mejora, un tipo de valor organizacional inclinado hacia el medio ambiente externo, que busca el control, la fijación de objetivos o la competitividad. 
Gráfica 2. Media de los valores organizacionales

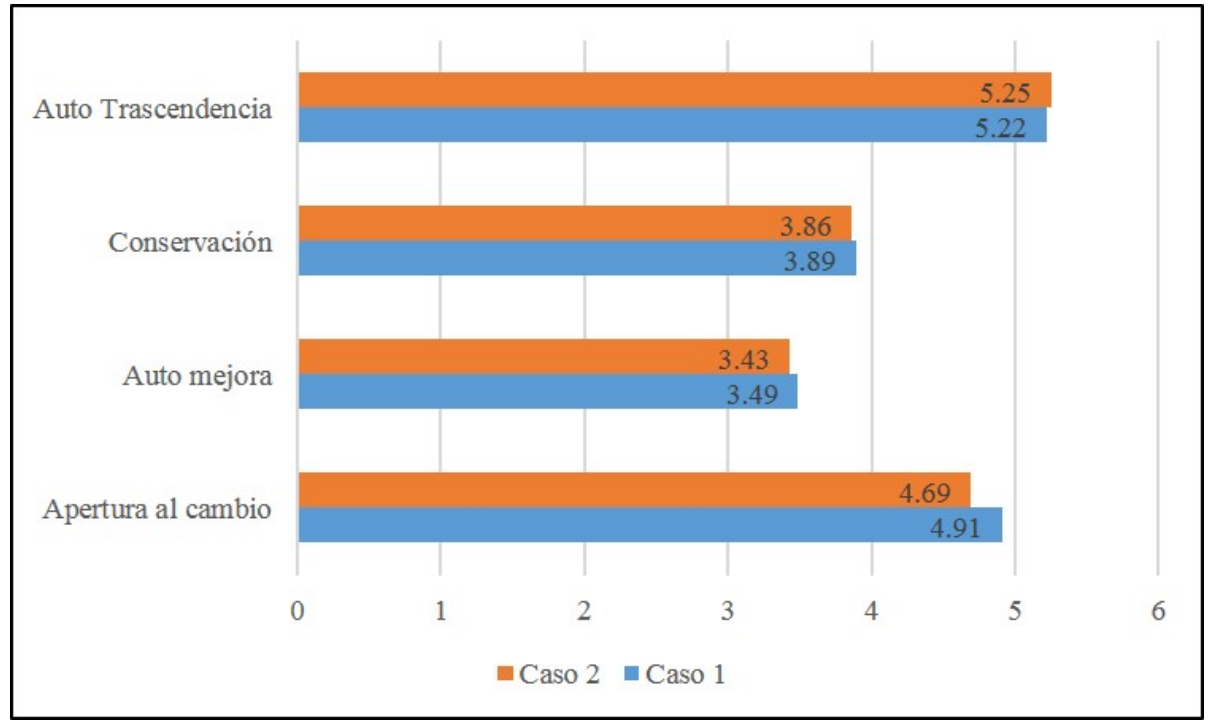

Fuente: elaboración propia.

Después se evaluaron cada una de las variables individuales, para ello se tomó el total de los participantes de los dos casos, analizando primero la variable edad, 57\% de la muestra eran trabajadores de entre 18 y 30 años, $32 \%$ de 31 a 40 años y $11 \%$ trabajadores de 41 años o más (gráfica 3).

Gráfica 3. Rangos de edad

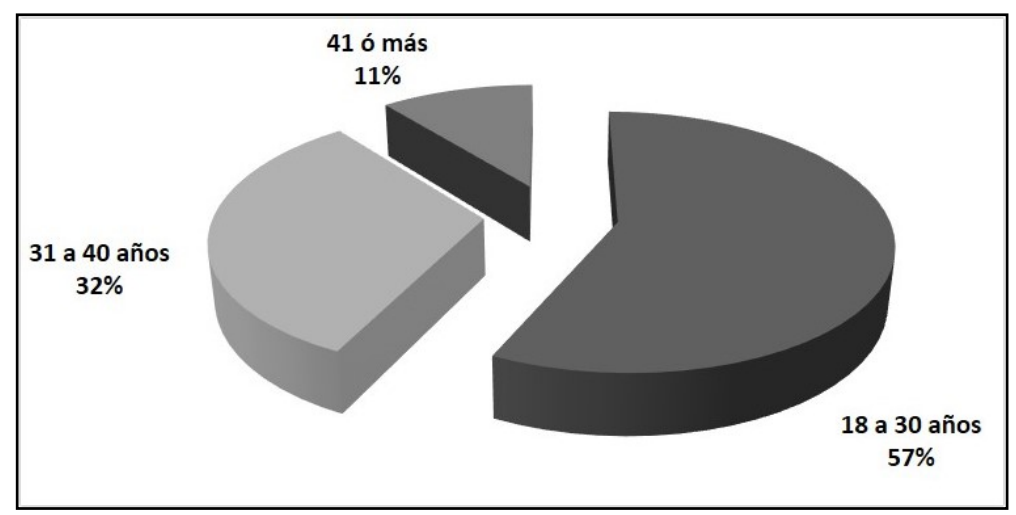

Fuente: elaboración propia. 
En cuanto a la variable edad y su relación con los valores organizacionales (gráfica 4), en los tres estratos de edad los trabajadores coincidieron con la clasificación, la tipología de valor más aceptada fue la de autotrascendencia.

\section{Gráfica 4. Edad y valores organizacionales}

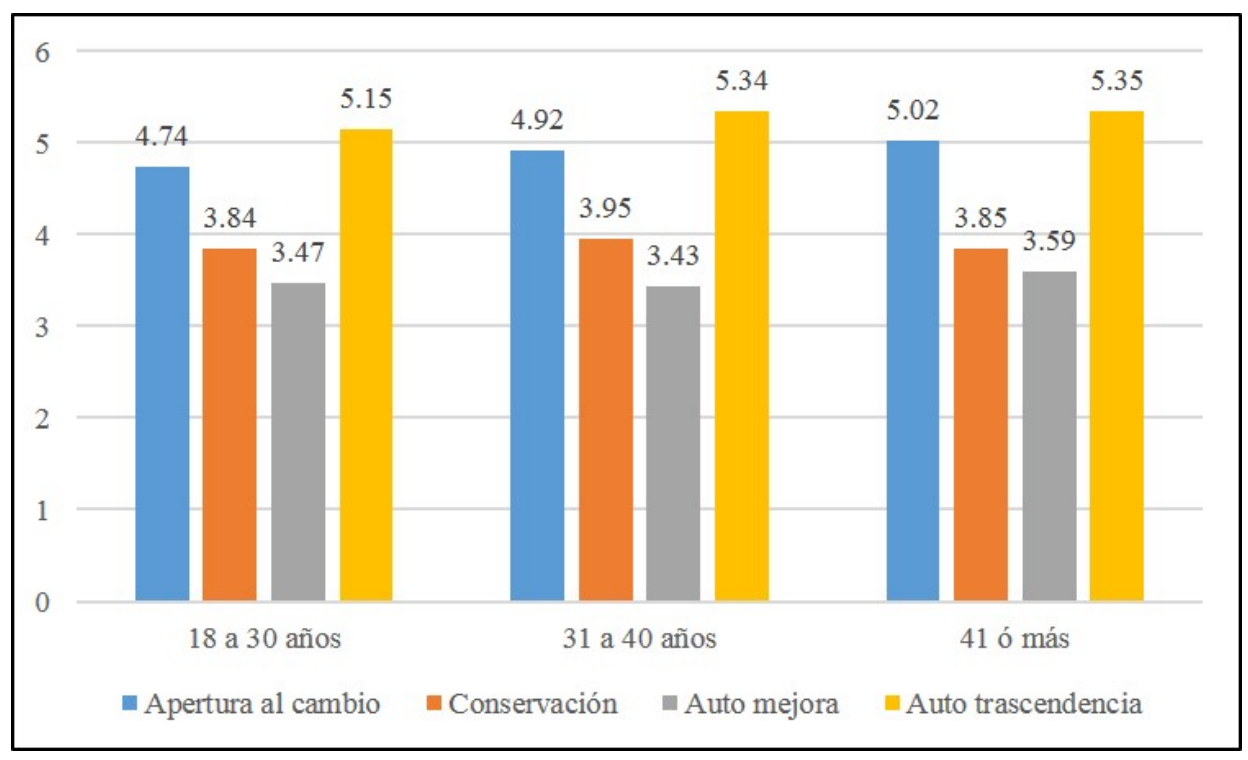

Fuente: elaboración propia.

Respecto a la variable género, en la encuesta participaron $167 \mathrm{mu}-$ jeres $(35 \%)$ y 306 hombres (65\%). Al evaluar el género y los valores organizacionales, el análisis arrojó resultados muy similares, en ambos casos se tuvo como el tipo de valor preponderante el de autotrascendencia (gráfica 5).

Al evaluar la variable estado civil de la muestra total obtenida (gráfica 6) se encontró que 130 individuos eran solteros (27\%), 292 casados (78\%) y 51 divorciados (11\%); se tuvieron resultados semejantes y la tipología de autotrascendencia fue a la que se dio más valía. 


\section{Gráfica 5. Género}

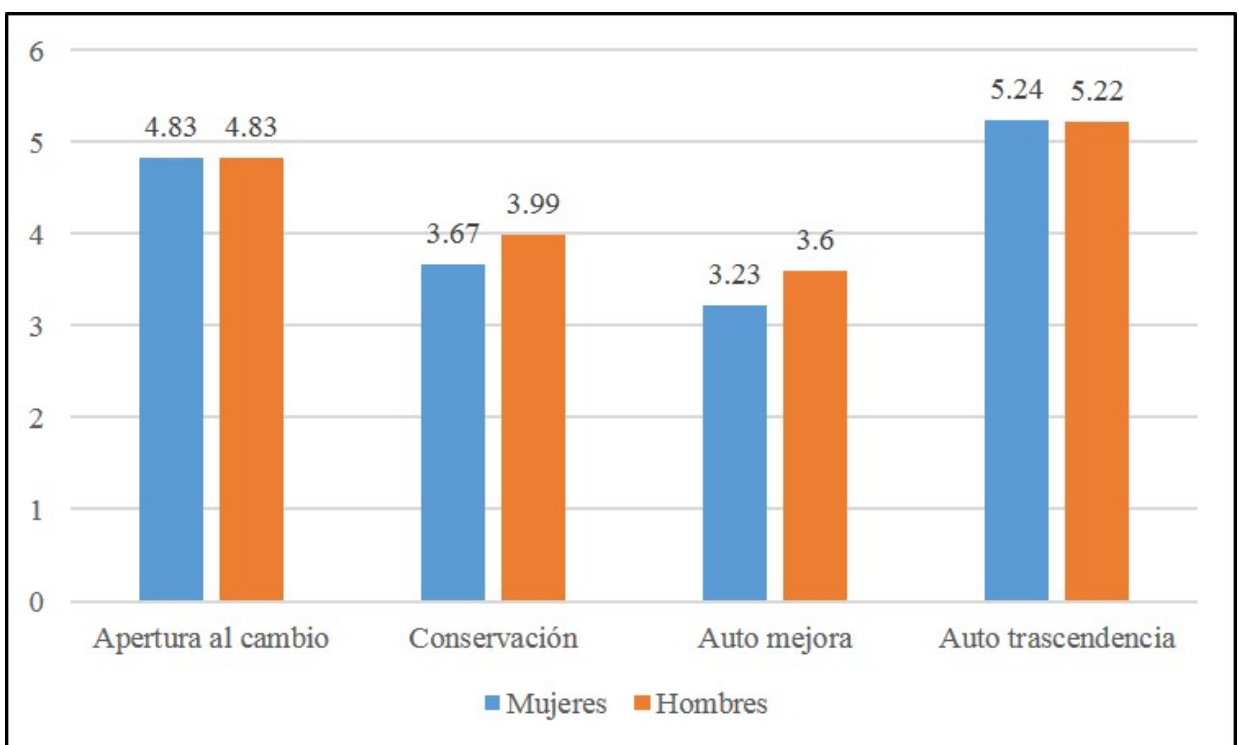

Fuente: elaboración propia.

Gráfica 6. Estado civil

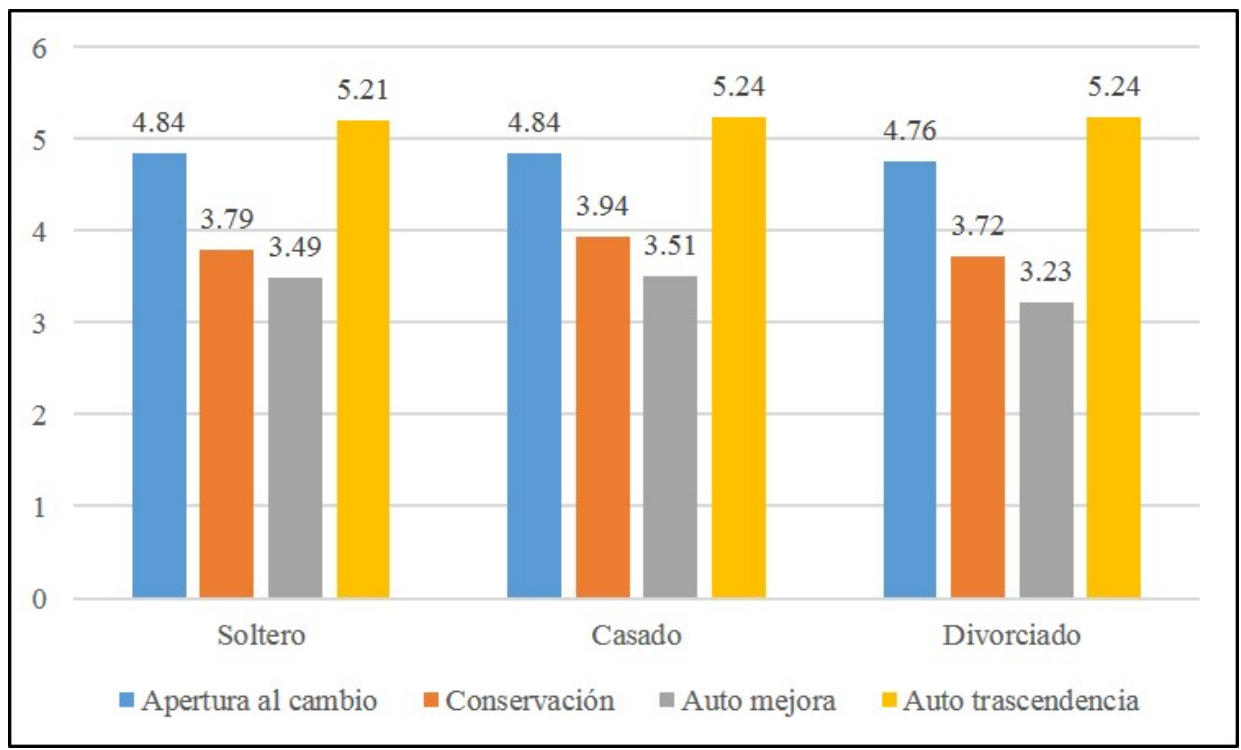

Fuente: elaboración propia. 
En la última comparación que se realizó, al analizar la variable antigüedad se encontró un comportamiento similar, la tipología a la que se ponderó más fue la de autotrascendencia (gráfica 7).

Gráfica 7. Antigüedad

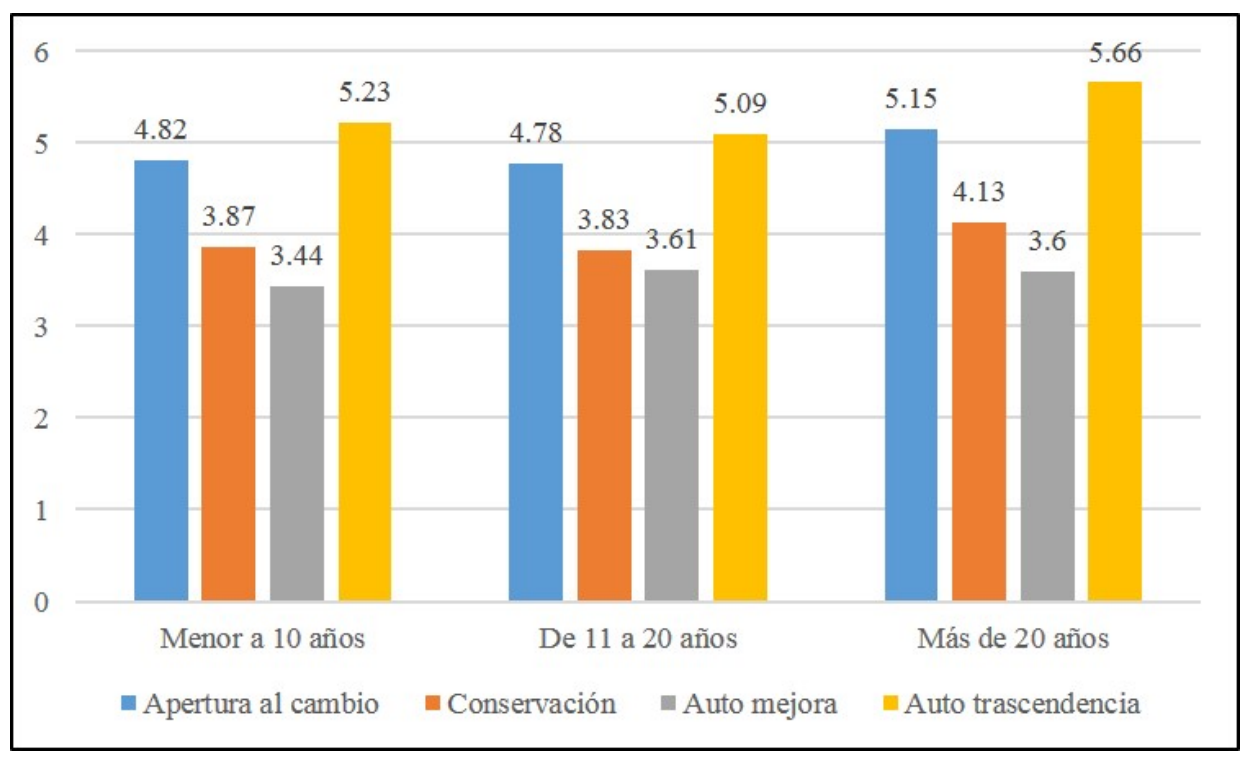

Fuente: elaboración propia.

Como puede apreciarse mediante los resultados del análisis univariante la mayoría de los individuos, independientemente de variables individuales como edad, género, estado civil o antigüedad, mostraron una mayor inclinación hacia la tipología de valores organizacionales de autotrascendencia.

\subsection{Análisis multivariante}

A la par del análisis univariante se efectuó un análisis multivariante con el objeto de generar información más profunda que permitiera explicar con mayor detalle el comportamiento de las variables estudiadas. 
En el cuadro 4 se muestran las estimaciones de las variables previamente mencionadas, se evalúan las relaciones entre los cuatro tipos de valores organizacionales (apertura al cambio, conservación, automejora, autotrascendencia) y las distintas variables demográficas (edad, género, estado civil y antigüedad).

Cuadro 4. Relación entre valores organizacionales y variables demográficas

\begin{tabular}{|c|c|c|c|c|c|}
\hline & & & & & \\
\hline & iable & Apertura & Conservación & Auto & Auto \\
\hline Edad & $<30$ años & 0.054 & 0.098 & 0.100 & 0.118 \\
\hline & 31- 40 años & 0.333 & 0.201 & 0.001 & 0.308 \\
\hline & $>40$ años & 0.188 & 0.906 & 0.925 & 0.776 \\
\hline Género & Masculino & 0.000 & 0.000 & 0.000 & 0.000 \\
\hline & Femenino & 0.000 & 0.000 & 0.000 & 0.000 \\
\hline Estado civil & Soltero & 0.000 & 0.000 & 0.002 & 0.000 \\
\hline & Casado & 0.000 & 0.000 & 0.000 & 0.000 \\
\hline & Divorciado & 0.670 & 0.574 & 0.048 & 0.326 \\
\hline Antigüedad & $<10$ años & 0.606 & 0.005 & 0.002 & 0.152 \\
\hline & $11-20$ años & 0.743 & 0.005 & 0.139 & 0.411 \\
\hline & $>20$ años & 0.633 & 0.452 & 0.332 & 0.609 \\
\hline
\end{tabular}

Fuente: elaboración propia.

\subsubsection{EFECTO DE LA EDAD EN LOS VALORES ORGANIZACIONALES}

Respecto a la variable edad y su relación con los distintos tipos de valores organizacionales, se encontró que en el caso de los trabajadores menores de 30 años no existe una relación significativa entre su edad y los cuatro tipos de valores. En este caso los coeficientes de contingencia son 
muy bajos (cuadro 5), el más alto es el relacionado con la variable autotrascendencia (0.118), lo cual indica una correlación prácticamente nula. En cuanto a los individuos con edad comprendida entre 31 y 40 años se observó una correlación baja, los valores organizacionales de apertura al cambio (0.333) y auto trascendencia (0.308) tuvieron las estimaciones más altas, aunque poco significativas. Por su parte, al analizar la relación entre los valores organizacionales y los individuos con edades mayores a 40 años, los resultados muestran mayor intensidad en la correlación con las variables automejora (0.925), conservación (0.906) y autotrascendencia (0.776), respectivamente. En los dos primeros casos se puede hablar de una correlación muy alta, mientras que en el tercer valor organizacional de una correlación alta.

Al hacer una comparación de estos resultados con los obtenidos por Arciniega (1999: 4) puede precisarse que existe cierta concordancia, ya que los trabajadores estudiados en la presente investigación mostraron una mayor predilección por los tipos de valores de auto mejora y conservación. Dichos valores, a pesar de tener ciertas diferencias, en el fondo se encuentran relacionados porque ambos tienen un enfoque hacia la estabilidad en el trabajo, lo cual se relaciona con lo indagado por Arciniega (1999: 4), quien menciona que las personas mayores de 40 años le dan una mayor significación a valores como conformidad o seguridad.

Cuadro 5. Coeficiente de correlación

\begin{tabular}{ll}
\hline \multicolumn{1}{c}{ Coeficiente } & \multicolumn{1}{c}{ Interpretación } \\
\hline De 0 a 0.20 & Correlación prácticamente nula \\
De 0.21 a 0.40 & Correlación baja \\
De 0.41 a 0.70 & Correlación moderada \\
De 0.71 a 0.90 & Correlación alta \\
De 0.91 a 1 & Correlación muy alta \\
\hline
\end{tabular}

Fuente: elaboración propia. 


\subsubsection{EFECTO DEL GÉNERO EN LOS VALORES ORGANIZACIONALES}

Respecto al género, el análisis de la información recabada para esta investigación en ningún caso mostró una correlación entre las variables individuales y los valores organizacionales de los individuos participantes. Este resultado, a pesar de ser desconcertante, tiene cierta coherencia si se toma en cuenta un estudio similar como el realizado por Abbu-Saad e Isralowitz (1997: 749), quienes encontraron correlaciones entre distintas variables individuales y los valores, aunque en el caso del género no se localizaron patrones sólidos que establecieran una relación.

\subsubsection{EFECTO DEL ESTADO CIVIL EN LOS VALORES ORGANIZACIONALES}

Al realizar un análisis acerca del estado civil y su relación con los valores organizacionales se encontró, por ejemplo, que en el caso de las personas solteras los coeficientes de contingencia fueron muy bajos, lo que puede interpretarse como una correlación nula. En el caso de las personas casadas se presentó la misma situación, ya que los coeficientes de contingencia tuvieron valores prácticamente nulos, por lo que no puede hablarse de una relación entre ambas variables. Respecto a las personas que manifestaron ser divorciadas, se observaron resultados que hablan de una mayor relación con los tipos de valores organizacionales. Por ejemplo, en el caso del valor apertura al cambio se obtuvo una estimación de 0.670 , que puede interpretarse como una correlación moderada, es posible que las personas divorciadas debido a lo experimentado en sus vidas se sientan identificadas con valores como la flexibilidad, individualidad o independencia.

En el caso de la relación entre personas divorciadas y el tipo de valor conservación se obtuvo una ponderación de 0.574 , lo cual habla también de una correlación moderada. Dicho resultado es contrastante, ya que el tipo de valor conservación es contrario al tipo de valor apertura al cambio, 
debido a que en la primera tipología se aprecian valores como la estabilidad, el control o la tradición. Posteriormente, en la relación del estado civil divorciado y el tipo de valor auto trascendencia se obtuvo una ponderación de 0.326 , lo que indica una correlación baja entre estas variables; en el caso del tipo de valor de auto mejora se observó una ponderación prácticamente nula con un índice de 0.048 , por lo que puede apreciarse, en ambos casos, que las personas divorciadas no están tan identificadas con este tipo de valores.

\subsubsection{EFECTO DE LA ANTIGÜEDAD EN LOS VALORES ORGANIZACIONALES}

Finalmente, en referencia a la relación entre la antigüedad en el trabajo y los tipos de valores organizacionales, se encontró que aquellas personas con una estancia igual o menor a diez años laborando en la organización muestran una mayor empatía con el valor de apertura al cambio (0.606), lo cual puede estar relacionado con la juventud o lo poco rutinario de su relación laboral, en una primera instancia. La apertura al cambio es una tipología de valor que tiene un enfoque hacia el medio ambiente externo, que busca la flexibilidad e individualidad, además de la innovación o la creatividad. Dichas características se relacionan con lo indagado por Abboushi (1990: 63), quien encontró que los trabajadores con menor tiempo en la organización pretenden demostrar un mayor interés en su trabajo con el objeto de asegurar su estancia dentro de la empresa, para lo cual se pueden servir de valores como los mencionados anteriormente.

Respecto a los trabajadores con una antigüedad oscilante entre 11 y 20 años se encontró que éstos tienen una mayor coincidencia con el valor de apertura al cambio (0.743), en comparación con los más jóvenes, lo que contrasta con los resultados obtenidos por Abboushi (1990: 63), quien menciona que los trabajadores con una antigüedad media presentaban un interés menor por demostrar su valía, quizá debido a que ya habían 
adquirido cierta estabilidad en su trabajo. En el caso de las empresas estudiadas, de dicho hallazgo se puede inferir que los trabajadores de antigüedad media, a pesar de tener cierta solidez gremial, aún conservaban el interés por mostrar una dinámica laboral.

Por último, en el caso de los trabajadores con una antigüedad mayor a 20 años, se encontró una mayor diversidad en los resultados, ya que estos le dieron una mayor importancia a las tipologías de apertura al cambio (0.633) y autotrascendencia (0.609), lo cual indica una correlación moderada. En ambos casos, dichas tipologías de valores coinciden en cuanto a que se identifican con la flexibilidad, aunque en el primer caso hay un mayor enfoque hacia el medio ambiente externo, a diferencia del segundo en el que su orientación es hacia las relaciones internas. Los resultados obtenidos difieren del estudio elaborado por Abboushi (1990: 63), quien establece que aquellos trabajadores con una antigüedad mayor a 20 años mostraban una mayor identificación con la conservación del estatus debido a la búsqueda de asegurar mejores condiciones al momento de retirarse. En el caso de las empresas estudiadas es probable que, debido a su alta competitividad, los trabajadores se encuentran en un proceso de constante cambio, por lo que no es posible la coincidencia con una tipología de valor como la de conservación, por ejemplo.

\section{CONCLUSIONES}

En la presente artículo pudo investigarse acerca de la influencia de las variables individuales en los valores organizacionales de los trabajadores de dos empresas manufactureras establecidas en el estado de Aguascalientes. Los resultados muestran que algunas variables individuales sí influyen significativamente en los valores organizacionales expresados por los trabajadores encuestados, con lo que se comprueba la hipótesis planteada para este estudio. En el caso de la edad de los trabajadores y su influencia en los tipos de valores organizacionales, ésta se da únicamente en aquellas personas mayores de cuarenta años. La información encontrada brinda 
evidencia significativa por cuyo medio se puede afirmar que la mayor edad sí está relacionada con tipologías de valores como la conservación o la automejora, las cuales aunque tienen un enfoque divergente, por un lado hacia el medio ambiente interno y por otro hacia el externo, en ambos casos buscan una estabilidad relacionada con la conformidad o seguridad reportada en otros estudios.

Respecto al género, al estudiar dicha variable y su relación con las tipologías de valores los resultados no muestran una correlación significativa, por lo que no puede hablarse de que el ser mujer u hombre tenga una relación directa con la empatía hacia algún valor organizacional en particular.

Por otro lado, en cuanto a la variable estado civil se encontró que únicamente en el caso de las personas divorciadas hay una correlación moderada con los valores de apertura al cambio y conservación, lo cual es contrastante, puesto que dichas tipologías de valores son opuestas y por lo tanto pretenden alcanzar objetivos diferentes. Por una parte, la tipología de apertura al cambio se enfoca hacia el medio ambiente externo y está de acuerdo con la flexibilidad, mientras que la tipología de conservación tiene un enfoque hacia el medio ambiente interno y a una estabilidad en el trabajo.

En cuanto a la antigüedad, los resultados muestran que sí hay una relación entre esta variable y los tipos de valores organizacionales; se encontró, por ejemplo, que en aquellos trabajadores con una antigüedad menor a diez años hay una correlación moderada con valor de apertura al cambio. Con lo anterior puede establecerse que las personas con un menor tiempo de estancia en la organización son individuos en su mayoría jóvenes, con una orientación hacia la flexibilidad, independencia o creatividad para satisfacer los requerimientos del medio ambiente externo. En el caso de aquellos individuos con una estancia en la organización de entre 11 y 20 años se encontró que éstos tienen una mayor identificación con el valor de apertura al cambio, ya que se presenta una correlación alta. Este hallazgo es muy interesante, pues muestra que aunque estas personas tienen más tiempo laborando en la empresa de igual manera 
siguen identificándose con un valor que pareciera exclusivo para personas más jóvenes.

Al analizar la información obtenida de las personas mayores de 40 años se descubrió que, de igual manera, las estimaciones más altas de correlación se encontraron relacionadas con el tipo de valor de apertura al cambio; sin embargo, en este caso también hubo una correlación media con el tipo de valor de auto trascendencia. Ambas tipologías se encuentran identificadas con valores como la flexibilidad, lo que expresa una identidad con las necesidades competitivas de las empresas. No obstante, la tipología de apertura al cambio se enfoca a satisfacer los requerimientos del medio ambiente externo; mientras que la de auto trascendencia propone una mayor preocupación por el mantenimiento de las relaciones internas de la organización, lo que propicia un ambiente de trabajo que favorezca el bienestar de los trabajadores.

Además de lo anterior, es preciso comentar que esta investigación cumplió con el objetivo de averiguar si variables individuales como la edad, género, estado civil o antigüedad tenían una relación con los tipos de valores organizacionales, encontrándose que, en varios casos, sí se puede hablar de una correspondencia. Los resultados aquí obtenidos pueden apoyar los esfuerzos de las organizaciones estudiadas o de algunas otras por entender de qué manera los valores organizacionales de los trabajadores secundan el comportamiento deseado y apropiado de las personas para la consecución de los objetivos de las empresas.

Por último, debe precisarse que los resultados de la presente investigación han sido presentados a las áreas de comunicación y recursos humanos de las organizaciones estudiadas. Dichos hallazgos han sido valorados y existe la pretensión de tomarlos en cuenta para el diseño de las estrategias empresariales que contemplen prácticas de trabajo acordes con los valores organizacionales de los trabajadores. Referente a futuros estudios, se tiene la pretensión de ampliar el análisis de los valores organizacionales en empresas extranjeras que lleven a cabo sus operaciones en el estado de Aguascalientes. 


\section{REFERENCIAS}

Abboushi, S. 1990. "Impact of individual variables on the work values of Palestian Arabs", International Studies of Management and Organizations, 20(3): 53-68.

Abbu-Saad, I. y R. Isralowitz. 1997. "Gender as a determinant of work values among university students in Israel", The Journal of Social Psychology, 137(6): 749-763.

Alducin, E. 2004. Los valores de los mexicanos, tomo V, Grupo Financiero Banamex, México.

Arciniega, L. 1999. Work values in México; a cross-national study in eight cities, presentado en Joint European Conference of the International Association for Cross-Cultural Psychology and the International Test Commission, Graz, Austria.

y H. Zazueta. 2010. Desarrollo de los valores en el trabajo, $1^{\mathrm{a}}$. edición, Trillas, México.

Bell-Laroche, D., S. Kerwin y J. MacLean. 2014. "The mediating influence of management by values in nonprofit sport organizations", Journal of Sport Management, 28: 646-656.

Cuevas, R. 2005. "La construcción de la ética de la empresa o ética de los negocios. Primera parte. Las formas a partir de la ética", Contaduría y Administración, 216: 119-141.

Devos, T., D. Spini y S. Schwartz. 2002. "Conflicts among human values and trust in institutions", Bristish Journal of Social Psychology, 41: 481-494.

Florea, L., Y. Cheung y N. Herndon. 2013. "For All Good Reasons: Role of Values in Organizational Sustainability”, J Bus Ethics, 114: 393-408.

Glew, D. 2009. "Personal values and performance in teams: an individual and team-level analysis", Small Group Research, 40(6): 670-693. 
Jin, G. y D. Drozdenko. 2010. "Relationships among perceived organizational core values, corporate social responsibility, ethics, and organizational performance outcomes: An empirical study of information technology professionals", Journal of Business Ethics, 92: 341-359.

Jintae, F. y S. Xiao. 2012. "Work values, job satisfaction and organizational commitment in China", The International Journal of Human Resource Management, 23(10): 2144-2162.

Lipovetsky, G. 1998. El crepúsculo del deber, $4^{\mathrm{a}}$ edición, Anagrama, Barcelona.

Nelson, R. y S. Gopalan. 2003. "Do organizational cultures replicate national cultures? isomorphism, rejection and reciprocal opposition in the corporate values of three countries", Organization Studies, 24: $1115-1151$.

Pascale, R. y A. Athos. 1981. The art of japanese management: Applications for american executives, Warner, Nueva York.

Posner, B. 2010. "Another look at the impact of personal and organizational values congruency", Journal of Business Ethics, 97: 535-541.

Rodríguez, C. 2012. Impacto de las estrategias empresariales en la cultura organizacional a través de los valores organizacionales en empresas del estado de Aguascalientes. Estudio de casos, Universidad Autónoma de Aguascalientes, tesis de doctorado en Ciencias Administrativas, Aguascalientes.

Rokeach, M. 1973. Understanding human values individual and societal, The Free Press, Nueva York.

Sagiv, L. y S. Schwartz. 2007. "Cultural values in organisations: insights for Europe”, European Journal International Management, 1(3) 176-190.

Schmeltz, L. 2014. "Identical or just compatible? the utility of corporate identity values in communicating corporate social responsibility", International Journal of Business Communication, 51(3): 234-258. 
Schwartz, S. 1994. "Are there universal aspects in the structure and contents of human values?", Journal of Social Issues, 50(4): 19-45. 1999. "A theory of cultural values and some implications for work", Applied Psychology: An International Review, 48(1): 23-47.

M. Ros y S. Surkiss. 1999. "Basic individual values, work values, and the meaning of work", Applied Psychology: An International Review, 48 (1), 49-71.

Stelzl, M. y C. Seligman. 2009. "Multiplicity across cultures: multiple national identities and multiple value systems", Organization Studies, 30: 959-973.

WebFinance, Inc. (s/f). Business Dictionary, 2012, http://www.businessdictionary.com. 